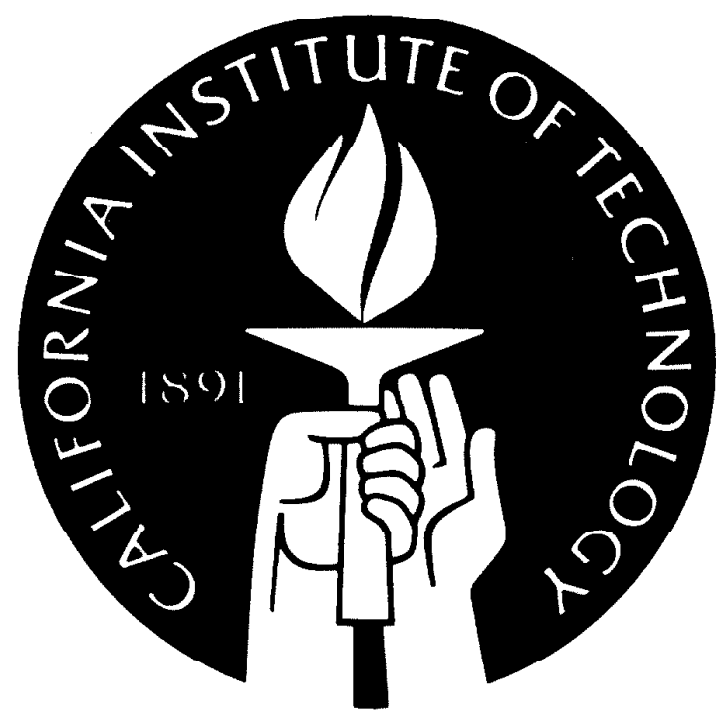

A Synthesis Method for Self-timed VLSI Circuits

Alain J. Martin

Department of Computer Science

California Institute of Technology

$5256:$ TR : 87 
A Synthesis Method for Self-Timed VLSI Circuits

Alain J. Martin

The research described in this paper was sponsored by the Defense Advauced Research Projects Agency, ARPA Order No. 6202, and monitored by the office of Naval Research under contract number N00014-87-K-0745

(C)California Institute of Technology, 1987

Department of Computer Science

California Institute of Technology

Pasadena, CA 91125

5256:TR:87

Published in: Proc. 1987 IEEE International Conference on

Design: VLSI in Computers 8 Processors

ICCD '87, Rye Town Hilton, Rye Brook, New York

October 5 - October 8, 1987; pp 224-229

IEEE Computer Society Press 


\section{A Synthesis Method for Self-timed VLSI Circuits}

Alain J. Martin

Department of Computer Science

California Institute of Technology

Pasadena CA 91125, USA

\section{Introduction}

With chip size reaching 1 million transistors, the need for highlevel design of circuits becomes compelling. The main stumbling block in the development of design methods for VLSI algorithms is to find an interface that provides a good separation of the physical and algorithmic concerns. Among the physical issues, timing is the most critical, since it is not only essential to the real-time behavior of a circuit, but also to its logical correctness if synchronous techniques are used.

Synchronous techniques are detrimental to the use of highlevel design methods because they don't "scale well": a circuit may cease to function correctly when its feature sizes are scaled down to smaller dimensions. Further. with the increasing size of circuits, it becomes more and more difficult to distribute safely a clock signal across a chip, and the restrictions attached to wire lengths in order to maintain certain timing properties add pxtra complication to the already difficult layout problem.

For all those reasons, self-timed techniques (as defined in [10]) are particularly attractive for high-level VLSI design [9]. We propose a synthesis method for self-timed circuits in which the computation is initially described as a set of communicating processes in the notation of [3], which is similar to C.A.R. Hoare's CSP [2] but augmented with the probe construct. This first description is the reference solution, which has to be proved correct. The program is then compiled into a self-timed circuit by applying a series of semantics-preserving transformations. Hence the circuit obtained is correct by construction.

Unlike most silicon compilation methods and hardware description languages, the method leads to efficient circuits. It has been applied with "hand compilation" to a series of difficult self-timed design problems, such as distributed mutual exclusion, fair arbitration, routing automata, with great success. Actually, the method, applied by a person in a mechanical way, will typically produce better results than the most experienced designers can produce. The main reason for the efficiency of the method is that, rather than going in one step from the program notation to the circuit, the designer applies a series of transformations to the original program. At each level of the transformation, powerful algebraic manipulations can be performed leading to important optimizations in terms of speed or area.

We shall first present the program notation and the VLSI operators that constitute the "object code". We then describe the four steps of the compilation and illustrate the method with one sizeable example, the construction of a stack. We shall conclude that this technique can be used for high quality and high complexity designs, fully automated from a provably correct high-level description. (For a more complete description of the method, see $[4],[5],[6]$, and $[7]$.
2. The program notation

The language used for the high-level description is close to C.A.R. Hoare's CSP[2]. We give only a very informal definition of the constructs used in this paper.

i) $b \uparrow$ stands for $b:=$ true,$b \downarrow$ stands for $b:=$ false

ii) The execution of the selection command (generalized IFstatement) $\left.\left|G_{1} \rightarrow S_{1}\right| \ldots \mid G_{n} \rightarrow S_{n}\right]$, where $G_{1}$ through $G_{n}$ are Boolean expressions, and $S_{1}$ through $S_{n}$ are program parts, $\left(G_{i}\right.$ is called a "guard", and $G_{i} \rightarrow S_{i}$ a "guarded command") amounts to the execution of an arbitrary $S_{i}$ for which $G_{i}$ holds. If $\neg\left(G_{1} \vee \ldots \vee G_{n}\right)$ holds, the execution of the command is suspended until $\left(G_{1} \vee \ldots \vee G_{n}\right)$ holds.

iii) For atomic actions $x$ and $y, ~ " x, y$ " stands for the execution of $x$ and $y$ in any order.

iv) $[G]$ where $G$ is a Boolean, stands for $[G \rightarrow$ skip], and thus

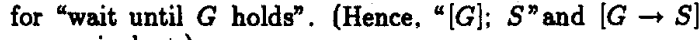
are equivalent.)

v) $*[S]$ stands for "repeat $S$ forever".

vi) From ii) and iii), the operational description of the statement $*\left[\left[G_{1} \rightarrow S_{2}|\ldots| G_{n} \rightarrow S_{n}\right]\right]$ is "repeat forever: wait until some $G_{i}$ holds; execute an $S_{i}$ for which $G_{i}$ holds".

\section{Communicating processes}

A concurrent computation is described as a set of processes communicating with each other by communication actions on channels (no shared variables). When no messages are transmitted, communication on a channel is reduced to synchronization signals. The name of the channel is then sufficient for identifying a communication action.

If two processes $p 1$ and $p 2$ share a channel named $X$ in $p 1$ and $Y$ in $p 2$, at any time the completion of the nth $X$. action "coincides" with the completion of the nth $Y$ - action. If, for example, $p 1$ reaches the nth $X$-action before $p 2$ reaches the nth $Y$-action, the completion of $X$ is suspended until $p 2$ reaches $Y$. The $X$-action is then said to be pending.

Probe

Instead of the usual selection mechanism by which a set of pending communication actions can be selected for execution, we provide a general Boolean command on channels, called the probe. In process $p 1$, the probe command $\bar{X}$ has the same value as the predicate "A communication action $Y$ is pending in $p 2$.

Hence the guarded command $\bar{X} \rightarrow X$ guarantees that the $X$-action is not suspended. And a construct of the form $[\bar{X} \rightarrow X \mid \bar{Y} \rightarrow Y]$ can be used for selection.

\section{The "object code"}

In standard digital VLSI design, the MOS transistor is idealized as an on/off switch. Unfortunately, the switch model is too crude, ignoring too many electrical phenomena that play 
an important role in the functioning of the circuit. Therefore, trying to carry the discrete model of a computation down to the transistor level is very likely to lead either to incorrect implementations or to a too complicated model of the computation. A crucial decision in the developement of our method has been to choose an "object code" at a higher level than the transistor. We have chosen to construct a notation that provides the weakest possible form of control structure and smallest number of program constructs. In fact, the notation contains exactly one construct, the production rule, and is therefore called the "production-rule set notation".

This minimal notation has been chosen so that i) it has sound semantics, ii) any non-terminating program can be compiled into production rules, ii) the transformation into a circuit is straightforward.

In fact, we consider the production-rule set as the canonical representation of a circuit. This representation can be decomposed into several equivalent networks of gates depending on the set of building blocks used, but the production-rule set represents the circuit independently of the gate implementations.

\section{4.' Production rules}

Production rules can be seen as a weaker form of guarded commands. Consider the production rule $G \mapsto S$

- $S$ is either a simple assignment, or an unordered list " $s 1$, $s 2, s 3, \ldots$ of simple assignments, where a simple assignment is the assignment of true or false to a single Boolean variable.

- $G$ is a Boolean expression, called the guard of the production rule. If $G$ holds, the correct execution of $S$ is guaranteed anly if $G$ remains invariantly true until the completion of $S$. We say that $G$ must be stable.

A production rule set is an unordered set (a collection) of production rules. Consider the canonical production rule set PRS:

$$
\begin{aligned}
G 1 & \mapsto S 1 \\
G 2 & \mapsto S 2 \\
& \ldots \\
G n & \mapsto S n
\end{aligned}
$$

- Unlike the guarded commands of a selection or a repetition, the mutual exclusion among the different production rules of a set is not part of the semantics of the construct. The correct execution of a production rule set is guaranteed only if interfering production rules are mutually exclusive. Two production rules are said to be interfering when their right-hand sides share a variable. Each process will be implemented as a p.r.s. such that exactly one p.r. is firable at any time, hence enforcing non-interference.

- If stability of the guards and mutual exclusion among inverfering production rules are guaranteed, whe production rule set $P R S$ is semantically equivalent to the non-terminating repetition *[[GCS]], where GCS is the guarded command set syntactically identical to PRS. Stability of the guards is essential to guaranlee the absence of races and hazards. When stability cannot be enforced, a special operator called "synchronizer" has to be used. When mutual exclusion cannot be enforced, a special operator called "arbiter" has to be used. These two operators are not needed in this paper.

We implement a p.r.s. by decomposing it into a collection of production rule sets each of which has a known VLSI implementation. Those primitive production rule sets correspond to logic gates or standard VLSI cells that are our ultimate building blocks._
The set of operators with which we want to build our circuits is not unique. The descriptions of the operators used in this paper in terms of their production rules and their logic symbols are as follows.

The "and":

$$
\begin{aligned}
&(x, y) \Delta z \equiv x \wedge y \mapsto z \uparrow \\
& \neg x \vee \neg y \mapsto z \downarrow .
\end{aligned}
$$

The "or":

$$
(x, y) \underline{\vee} z \equiv x \vee y \mapsto z \uparrow
$$

$$
\neg x \wedge \neg y \mapsto z \downarrow .
$$

The wire:

$$
x \underline{w} y \equiv x \mapsto y \uparrow
$$

$$
\neg x \mapsto y \downarrow .
$$

The fork:

$$
\begin{aligned}
x \underline{f}(y, z) \equiv & x \mapsto y \uparrow, z \uparrow \\
& \neg x \mapsto y \downarrow, z \downarrow .
\end{aligned}
$$

The C-element:

$$
\begin{aligned}
&(x, y) \underline{C} z \equiv x \wedge y \mapsto z \uparrow \\
& \neg x \wedge \neg y \mapsto z \downarrow .
\end{aligned}
$$

The asymmetric C-element:

$$
\begin{aligned}
(x ; y) \underline{a C} z \equiv & x \wedge y \mapsto z \uparrow \\
& \neg x \mapsto z \downarrow
\end{aligned}
$$

The "flip-flop":

$$
(x ; y) \underline{f f} z \equiv \begin{aligned}
& x \mapsto z \uparrow \\
& y \mapsto z \downarrow
\end{aligned}
$$

A negated input or output is represented on the figures by a small circle on the corresponding port. A wire with its input negated is an inverter. A cell with a negated input is considered as one cell, and not as the composition of an inverter and a cell.

\section{The compilation method}

Process decomposition

The first step of the compilation, called "process decomposition", consists in replacing a process by several semantically equivalent processes. The purpose of the decomposition is to obtain a process representation of the program in which the right-hand side of each guarded command is a straight-line program, i.e., consists only of simple assignments and communication commands, composed by semi-colons and commas. Process decomposition is applied repeatedly until the right-hand side of each guarded command is a straight-line program. Process decomposition plays an important role in the compilation of large programs. We won't need it in the example treated here. See [5] for a typical use of this transformation.

\section{Handshaking expansion}

The implementation of communication, called "handshaking expausion", replaces each chanuel by a pair of wire-uperators and each communication action by its implementation in terms of a "four-phase handshaking" protocol. Channel $(X, Y)$ is implemented by the two wires (xo $\underline{w} y i)$ and (yo $\underline{w} x i)$.

Initially, $x o, x i, y o$, and $y i$ are false. For a matching pair $(X, Y)$ of actions, the implementation is not symmetrical in $X$ and $Y$. One action is called active and the other one passive. The four-phase implementation with $X$ active and $Y$ passive is:

$$
X \equiv x \circ \uparrow ;[x i] ; x \circ \downarrow ;[\neg x i]
$$




$$
Y \equiv[y i] ; \text { yo } \uparrow ;[\neg y i] ; \text { yo } \downarrow
$$

When no action of a matching pair is probed, the choice of which one should be active and which une passive is arbitrary, but a choice has to be made. The choice can be important for the composition of identical circuits. A simple rule is that for a given channel $(X, Y)$, all actions at one side are active and all octions at the other side are passive. If $\bar{X}$ is used, all $X$ - actions are passive- with the obvious restriction that $\bar{Y}$ cannot be used in the same program. The implementation of the probe is simply:

$$
\begin{aligned}
& \bar{X} \equiv x i \\
& \bar{Y} \equiv y i
\end{aligned}
$$

A probed communication action $\bar{X} \rightarrow \ldots X$ is implemented:

$$
x i \rightarrow \ldots x o \uparrow ;[\neg x i] ; x o \downarrow
$$

\section{Reshuffling}

Consider the handshaking expansion of program $p$ according to (1), (2), and (3). Provided that the cyclic order of the four handshaking actions of a communication command is respected, the last two actions of this command can be inserted at any place in $p$ without invalidating the semantics of the communication involved. However, modifying the order of these two actions relatively to other actions of $p$ may introduce deadlock. The possibility to reshuffle the sernnd half of the handohalking sequence, plays an important role in the compilation method as a source of algebraic manipulations.

\section{Production rule expansion}

The uext slep is to compile the handshaking expansion of the program into a set of production rules from which all explicit sequencing has been removed. This is the most difficult step in particular because it requires, in all but trivial cases, the introduction of state variables to identify each state of the computation uniquely.

\section{Operator reduction}

The last step, called "operator reduction", consists in identifying sets of production rules in the program with sets of production rules describing operators. The non-trivial part in this step is called "symmetrization". It is used for transforming the guards of the production rules so as to make them 'look like' the guards of operators. After this last step, the program has been replaced by a network of operators for which standard cells exist. (We have constructed a cell library of self-timed elements in SCMOS technology. Since many cells are parametrized, the library is extendable.)

\section{Example: single variable register}

Consider the following process that provides read and write access to a simple boolean variable $x$ :

$$
*[[\bar{P} \rightarrow P ? x \mid \bar{Q} \rightarrow Q ! x]]
$$

where $\neg \bar{P} \vee \neg \bar{Q}$ holds at any time, i.e., read and write requests exclude each other in time.

Handshaking expansion

The handshaking expansion of (4) uses the "double-rail" technique: the Boolean value of $x$ is encoded on two wires, one

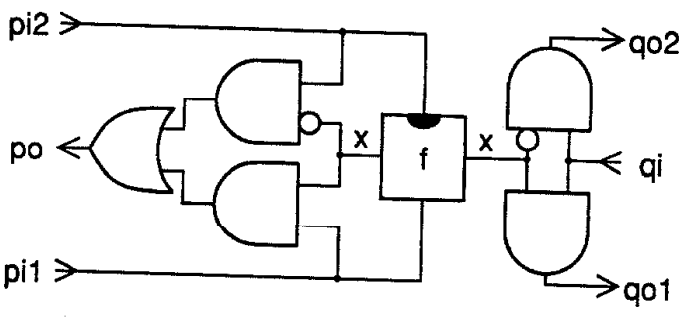

Figure 1: Single-bit register

for the value true and one for the value false. Each guarded command of (1) is expanded to two guarded commands:

$$
\begin{aligned}
& *\left[\left[p i_{1} \rightarrow x \uparrow ;[x] ; \text { po } \uparrow ;\left[\neg p i_{1}\right] ; \text { po } \downarrow\right.\right. \\
& \mid p i_{2} \rightarrow x \downarrow ;[\neg x] ; \text { po } \uparrow ;\left[\neg p i_{2}\right] ; p o \downarrow \\
& \mid x \wedge q i \rightarrow q o_{1} \uparrow ;[\neg q i] ; q o_{1} \downarrow \\
& \mid \neg x \wedge q i \rightarrow q 0_{2} \uparrow ;[\neg q i] ; q o_{2} \downarrow \\
& ]] .
\end{aligned}
$$

Production rule expansion The production-rule expansion of the first two guarded commands gives:

$$
\begin{aligned}
p i_{1} & \mapsto x \uparrow \\
p i_{1} \wedge x & \mapsto p o \uparrow \\
\neg p i_{1} & \mapsto p o \downarrow \\
p i_{2} & \mapsto x \downarrow \\
p i_{2} \wedge \neg x & \mapsto p \circ \uparrow \\
\neg p i_{2} & \mapsto p o \downarrow
\end{aligned}
$$

The first and fourth p.r.'s correspond to the flip-flop: $\left(p i_{1} ; p i_{2}\right) \underline{f} x$. The other p.r.'s can be transformed into:

$$
\begin{aligned}
\left(p i_{1} \wedge x\right) \vee\left(p i_{2} \wedge \neg x\right) & \mapsto p o \uparrow \\
\left(\neg p i_{1} \vee \neg x\right) \vee\left(\neg p i_{2} \vee x\right) & \mapsto p o \downarrow
\end{aligned}
$$

which is the definition of the IF-cell $\left(p i_{1} ; p i_{2} ; x\right) I F p o$. This set of p.r.'s can also be implemented as:

$$
\begin{aligned}
& \left(p i_{1}, x\right) \wedge p o_{1} \\
& \left(p i_{2}, \neg x\right) \triangle p o_{2} \\
& \left(p o_{1}, p o_{2}\right) \backslash p o .
\end{aligned}
$$

The production-rule expansion of the last two guarded commands of (5) gives:

$$
\begin{aligned}
x \wedge q i & \mapsto q o_{1} \dagger \\
\neg x \vee \neg q i & \mapsto o_{1} \downarrow \\
\neg x \wedge q i & \mapsto q o_{2} \uparrow \\
x \vee \neg q i & \mapsto q o_{2} \downarrow
\end{aligned}
$$

which corresponds to the two operators $(x, q i) \Delta q 0_{1}$ and $(\neg x, q i) \triangle q o_{2}$. The circuit is represented in Figure 1 .

\section{The lazy stack}

A lazy stack is one in which the full elements, i.e., the elements of the stack that contain a piece of data, are not necessarily contiguous. For instance, after a "pop" operation removes a data portion from the top clement of the slack, the hole created in the top element is not filled even if some other element of the stack contains a data portion. Obviously, we must record 
whether a stack element is full or empty. In the implementation given in [3], a Boolean variable is used for this purpose. Here we shall use a different coding: a stack element is described as two programs-one for the empty case, one for the full case-which call each other in a mutually recursive way.

We restrict ourselves to Boolean data portions. A data portion is added to a stack element by a command on the input channel "in". A data portion is removed from a stack element by a command on the output channel "out". We assume that the environment never attempts to add portions to a full stack nor to remove portions from an empty stack. Hence a request to remove a portion from an empty stack causes the element to obtain the next data portion from the "rest of the stack". Such an artion uses the input channel "get". Similarly, a request to add a portion to a full element causes the element to push the portion it contains to the "rest of the stack". Such an action uses the output channel "put".

The program for the empty stack element is called $E$. The program for the full stack element is called $F$. We have

$$
\begin{aligned}
& E \equiv \overline{\text { in }} \rightarrow \text { in? } x ; F \quad F \equiv[\overline{\text { in }} \rightarrow \text { put } ! x ; \text { in } ? x ; F \\
& \overline{\text { out }} \rightarrow \text { get } ? x ; \text { out } ! x ; E \quad \mid \overline{\text { out }} \rightarrow \text { out } ! x ; E \\
& 1 . \\
& \text { ]. }
\end{aligned}
$$

The initialization of an empty stack element is a call of $E$. The initialization of a full stack element is a call of $\boldsymbol{F}$.

\section{Implementation of the control part}

Let us first implement the "control part" of the program, i.e., the programs $E$ and $F$ from which message communication has been removed. We assume that the stack is empty initially. Instead of using mutual recursion, we use (what may look like) a slightly less symmetrical coding of (6): we introduce the channel $\left(t, t^{\prime}\right)$ and call $F$ from within $E^{\prime}$ by the usual construction of process decomposition. We get

$$
\begin{array}{cc}
E \equiv *[[\overline{\text { in }} \rightarrow \text { in } ; t & F \equiv *\left[\left[\overline{t^{\prime}} \wedge \overline{\text { in }} \rightarrow \text { put } ;\right.\right. \text { in } \\
\mid \overline{\text { out }} \rightarrow \text { get } ; \text { out } & {\left[\overline{t^{\prime}} \wedge \overline{\text { out }} \rightarrow \text { out } ; t^{\prime}\right.} \\
\text { ]]. } & ]] .
\end{array}
$$

In the handshaking expansion, the choice of active and passive communications is entirely dictated by the occurrence of the probes. We get

$$
\begin{aligned}
& E \equiv \\
& *[[\neg \text { ti } \wedge \text { ini } \rightarrow \text { ino } \uparrow ;[\neg \text { ini }] ; \text { ino } \downarrow ; \text { to } \uparrow ;[\text { ti }] ; \text { to } \downarrow \\
& \mid \neg t i \wedge \text { outi } \rightarrow \text { geto } \uparrow ;[\text { geti }] ; \text { geto } \downarrow ;[\neg \text { geti }] ; \text { outo } \uparrow ;[\neg \text { outi }] ; \text { outo } \downarrow \\
& ]] \\
& F \equiv \\
& *\left[\left[t i^{\prime} \wedge \text { ini } \rightarrow \text { puto } \uparrow ;[\text { puti }] ; \text { puto } \downarrow ;[\neg \text { puti }] ; \text { ino } \uparrow ;[\neg \text { ini }] ; \text { ino } \downarrow\right.\right. \\
& \mid \text { ti }{ }^{\prime} \wedge \text { outi } \rightarrow \text { outo } \uparrow ;[\neg \text { outi }] ; \text { outo } \downarrow ; \text { to }^{\prime} \uparrow ;\left[\neg t i^{\prime}\right] ; \text { to } \downarrow \\
& \|] .
\end{aligned}
$$

\section{Compilation of $E$}

The first guarded command of $E$ is a standard passive-active buffer element implemented as an active-active buffer composed with a passive-passive adaptor (Fig. 2.a). The second guarded

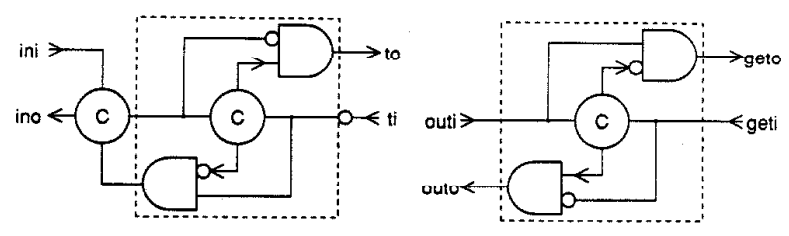

Figure 2: The two guarded commands of $E$

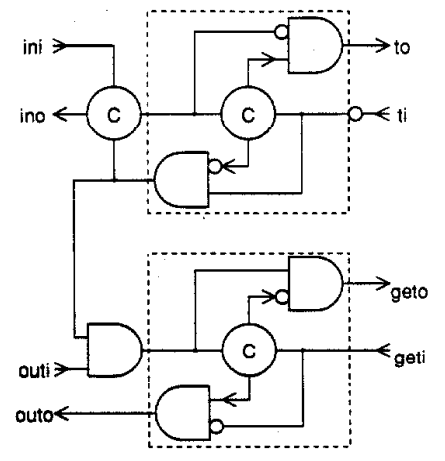

Figure 3: Implementation of $\mathrm{E}$

command is a standard stack element implemented as an activeactive buffer with input outi inverted (Fig. 2.b). The activeactive buffer is a standard cell called a $D$-element.

Next, we have to enforce mutual exclusion between the two guarded commands of $E$. Since in and out are mutually exclusive, it suffices to guarantee that when in is completed in the first guarded command, the second guarded command cannot start until $t$ is completed. In order to strengthen the guard of the second command with the appropriate expression, we introduce in the handshaking expansion of the first guarded command the variable $z$. We get

$z \wedge$ ini $\rightarrow$ ino $\uparrow_{i} z \downarrow ;$ [ $\neg$ ino $]$; ino $\downarrow ;$ to $\uparrow_{i}[t i] ;$ to $\left.\downarrow ; \mid \neg t i\right] ; z \uparrow$

as the handshaking expansion of the first guarded command. Obviously, it suffices to strengthen the guard of the second guarded command with $z$ to guarantee mutual exclusion between the two g.c.'s. We get

outi $\wedge z \rightarrow$ geto $\uparrow ;$ [geti]; geto $\downarrow ;[\neg$ geti]; outo $\uparrow ;[\neg o u t i] ;$ outo $\downarrow$

Since we can weaken $\neg$ outi as $\neg$ out $i \vee \neg z$, the only transformation is the replacement of out $i$ by $z \wedge$ outi. This gives the circuit of Figure 3 as an implementation of $E$.

\section{Compilation of $\mathbf{F}$}

The compilation of the first guarded command of $F$ is identical to that of the second command of $E$, with the appropriate change of variables. The compilation of the second command, however, can be drastically simplified by reshuffling. Since channel $\left(t, t^{\prime}\right)$ is an internal channel, we can reshuffle the handshaking sequence of $t^{\prime}$ without deadlock. The handshaking expansion of the second guarded command becomes:

$$
t^{\prime} i^{\prime} \wedge \text { outi } \rightarrow \text { outo } \uparrow ; t^{\prime} \uparrow ;\left[\neg t^{\prime} \wedge \text { ᄀouti }\right] ; \text { outo } \downarrow ; t^{\prime} \downarrow
$$

This sequence compiles immediately into the C-element: $\left(t^{\prime}\right.$, outi) $\underline{C}$ (outo, to') 


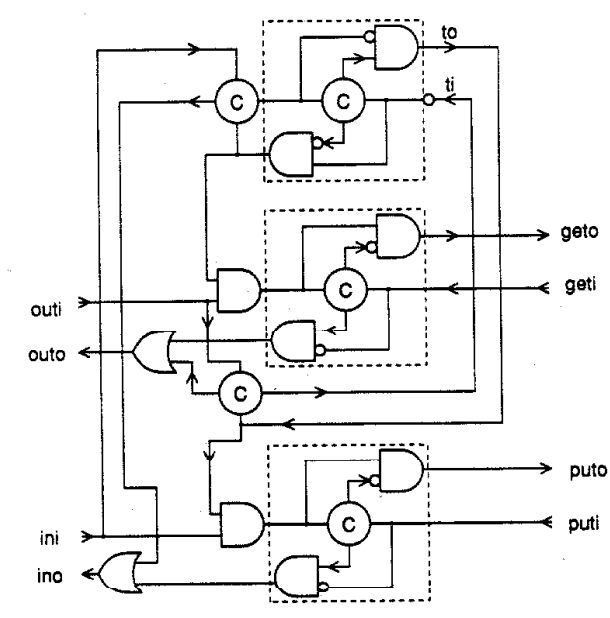

Figure 4: The control part of stack element

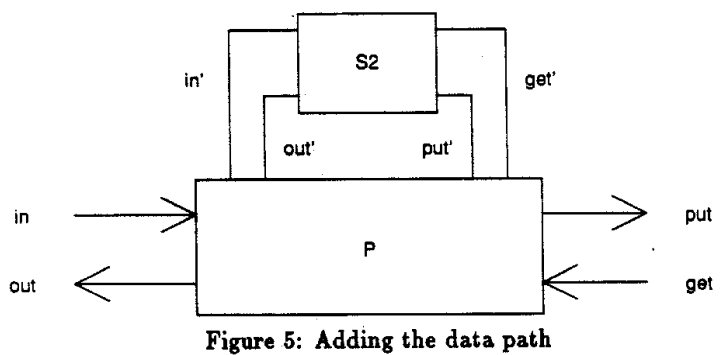

The channels in and out are used both in $E$ and $F$, so we need to merge the lucal cupies of in and the local copies of out in the standard way. The resulting circuit for the control part of the stack element is shown in Figure 4.

11. Implementation of the data path

Let $S 1$ and $S 2$ denote program (6) and program (7), respectively. We now have to extend the implementation of $S 2$ so as to obtain an implementation of $S 1$. We want to leave $S 2$ unchanged and introduce an extra "data path" process $P$ such that the parallel composition of $S 2$ and $P$ implements $S 1$. More precisely, the channels in, out, get, put of $S 2$ are renamed in', out', get', put'. $P$ communicates with $S 2$ via the renamed channels and with the environment via in, out, get, put. (See Figure 5).

By comparing $S 1$ and $S 2$, we derive that $P$ has to implement the operations:

$$
\begin{aligned}
& \text { in' } \bullet \text { in?x } \\
& \text { out' out!x } \\
& \text { get' } \bullet \text { get?x } \\
& \text { put' } \bullet \text { put!x }
\end{aligned}
$$

where $A \bullet B$ denotes the simultaneous execution of $A$ and $B$. (We can define the completion of an action so that the simultaneous execution of two actions is well-defined. The implementation of $A \cdot B$ amounts to interleaving the handshaking sequences of $A$ and $B$.)

The implementation of the four actions of $P$ is based on the register program constructed in Section 6 . For the sake of

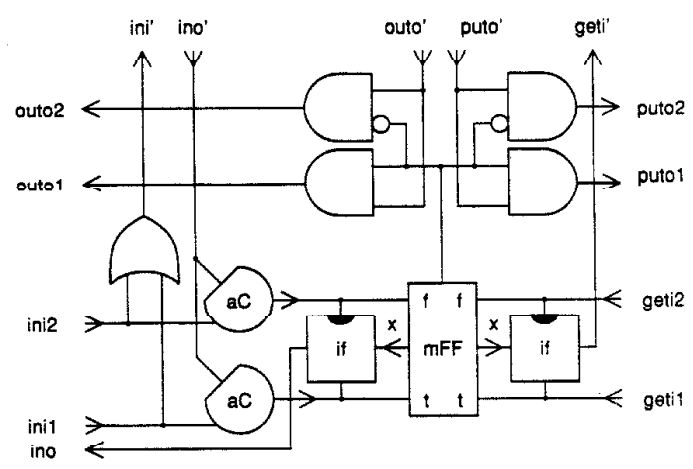

Figure 6: The data path

brevity, we omit the rest of the derivation which can be found in [8]. The entire data path is described in Figure 6.

The dual-port flip-flop used in the data path is defined as:

$$
\begin{aligned}
(s 1, s 2 ; t 1, t 2) \underline{2 f f} x \equiv s 1 \vee s 2 & \mapsto x \uparrow \\
t 1 \vee t 2 & \mapsto x \downarrow
\end{aligned}
$$

(By definition, at most one input is true at any time.)

12. The complete circuit

Two important optimizations are added to the design. The first one concerne the implementation of the second guard of $E$;

$$
\overline{\text { out }} \rightarrow \text { get? } x \text {; out } ! x .
$$

We obocrve that, in this case, unlike all other guarded com mands of (6), the value of $x$ involved in the second action (out $: x$ ) is the same as the value of $x$ involved in the first action (get? $\mathrm{x}$ ). We can therefore encode the value of $x$ in the handshaking expansion of the guarded command without having to use the register. The reshuffled handshaking expansion including the double-rail encoding of $x$ gives:

$$
\begin{aligned}
& \neg t i \wedge \text { outi } \rightarrow \text { geto } \uparrow ;[\text { geti } 1 \rightarrow \text { outo } 1 \uparrow \mid \text { geti } 2 \rightarrow \text { outo } \uparrow \uparrow] ; \\
& {[\text { outi] }] \text { geto } \downarrow ;[\neg \text { geti } 1 \rightarrow \text { outo } 1 \mid \neg \text { geti } 2 \rightarrow \text { outo } 2 \downarrow]}
\end{aligned}
$$

The circuit is

$$
\begin{aligned}
& (\neg t i, \text { outi }) \wedge \text { geto } \\
& \text { geti1 } 1 \text { w outo } 1 \\
& \text { geti } 2 \underline{w} \text { outo } 2
\end{aligned}
$$

The second optimization concerns the implementation of in' $\bullet$ in? $x$, which is more complex than that of get' $\bullet$ get? $x$ because in is passive while get is active. We replace in? $x$ and put! $x$ by ins;in? $x$ and outs; out! $x$, respectively, with ins passive and in active, and ouls aclive and out passive. For the output action out, the implementation is the same whether the channel is active or passive. The complete circuit is shown in Figure 7 with the data path extended to four bits.

\section{Concluding remarks}

By combining control and data, the design of a lazy stack encompasses most self-timed design issues (except for arbitration which is treated in [4] and [5]).

Let us summarize the main advantages of the method. First, the source language, in particular the use of the probe, 
produces compact and efficient algorithms, which can be further "tuned" through process decomposition. Second, the handshaking expansion combined with reshuftling offers powerful algebraic manipulations. Third, the production rule notation provides a canonical representation of the circuit which is straightforward to translate in whatever set of VLSI gates is available or convenient to use. Finally, the notion of stability of a guard captures exactly the necessary and sufficient condition to avoid races and hazards.

We already have a compiler that produces about the same design fully automatically [1]. Figure 8 shows a typical layout produced by the assembler from the operator set. Each operator has a standard cell representation. The cells of a process are stacked to form a tower in which power, reset, and ground run vertically.

ACKNOWLEDGEMENTS are due to Steve Burns for his contribution to the design of the stack, and to Cal Jackson for his help in the preparation of the manuscript. REFERENCES

[1] Burns, S., "Automated Compilation of Concurrent Programs into Self-timed Circuits". Caltech Computer Science Master's Thesis, in preparation, (1987).

[2] Hoare, C.A.R., "Communicating Sequential Processes". Comm. $A C M$ 21, 8, pp. 666-677 (August 1978).

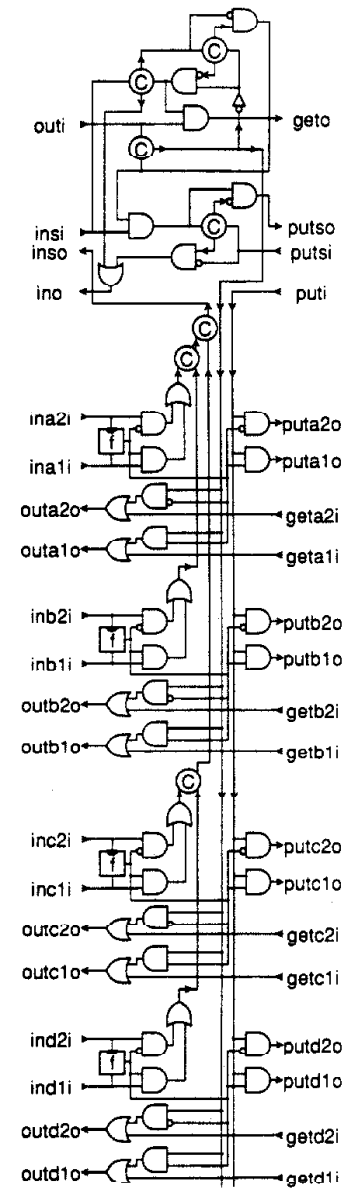

Figure 7: Stack element with four-bit data path
[3] Martin, A.J., "The Probe: an Addition to Communication Primitives", Information Processing Letters 20, pp. 125130 (1985).

[4] Martin, A.J., "The Design of a Self-Timed Circuit for Distributed Mutual Exclusion", Proc. 1985 Chapel Hill Conference on VLSI, ed. Henry Fuchs, pp. 247-260 (1985).

[5] Martin, A.J., "A Delay-Insensitive Fair Arbiter", Caltech Computer Science Technical Report 5193:TR:85 (1985).

[6] Martin, A.J., "Compiling Communicating Processes into Delay Insensitive VLSI circuits", in Distributed Computing, vol. 1, no 3, 1986.

[7] Martin, A.J., "Self-timed FIFO: an Exercise in Compiling Programs into Circuits" in From HDL description to ouaranteed correct circuit design, North-Holland, ed. Dnminique Borrione (1986).

[8] Martin, A.J., "Implementation of Communication with Message-passing", Caltech Computer Science Technical Report 5245:TR:87 (1987).

[9] Mead, C. and L. Conway, Introduction to VLSI Systems, Addison-Wesley, Reading MA (1980).

[10] Seitz, C.L., "System Timing", Chapter 7 in Mead \& Conway, Introduction to VLSI Systems, Addison-Wesley, Reading MA (1980).

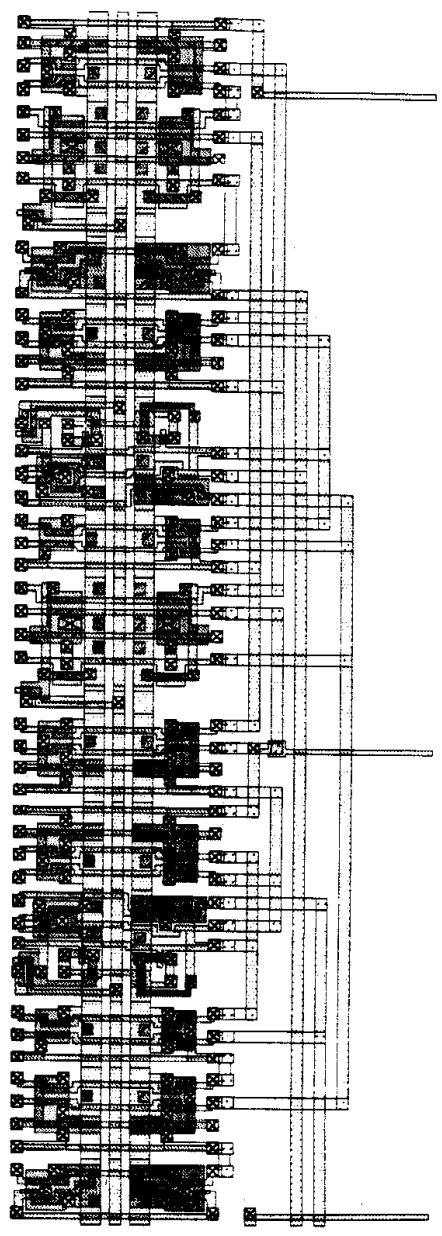

Figure 8: Layout of the control part 